\title{
A Novel function for Cactus/IкB inhibitor to promote Toll signals in the Drosophila embryo
}

Maira Arruda Cardoso ${ }^{1,4}$, Marcio Fontenele ${ }^{1,2}$, Bomyi Lim³ ${ }^{3}$ Paulo Mascarello Bisch

Stanislav Shvartsman ${ }^{3}$, Helena Marcolla Araujo $0^{1,2,5}$

${ }^{1}$ Instituto de Ciências Biomédicas, Federal University of Rio de Janeiro

${ }^{2}$ Institute of Molecular Entomology

${ }^{3}$ Lewis-Sigler Institute for Integrative Genomics, Princeton University, Princeton, NJ

${ }^{4}$ Instituto de Biofísica Carlos Chagas Filho, Federal University of Rio de Janeiro

${ }^{5}$ Corresponding author: Helena Araujo

Running title: Cactus favors Toll

\section{Summary:}

The IкB protein Cactus favors high Toll signals, revealing that the ventral and lateral aspects of the Dorsal/NFKB nuclear gradient behave differently respective to Cactus concentrations in the Drosophila embryo.

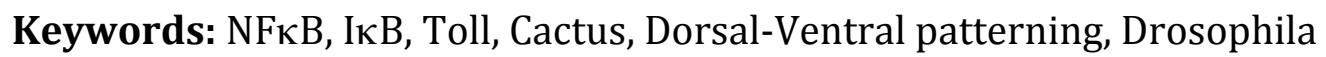

Word count: 2924 words

\begin{abstract}
The evolutionarily conserved Toll signaling pathway controls innate immunity across phyla and embryonic patterning in insects. In the Drosophila embryo Toll is required to establish gene expression domains along the dorsal-ventral axis. Pathway activation induces degradation of the IкB inhibitor Cactus resulting in a
\end{abstract}


nuclear gradient of the NFKB effector Dorsal. Here we investigate how cactus modulates Toll signals through its effects on the Dorsal gradient and Dorsal target genes. Quantitative analysis using a series of loss and gain-of-function conditions shows that the ventral and lateral aspects of the Dorsal gradient behave differently respective to Cactus fluctuations. Unexpectedly, Cactus favors Dorsal nuclear localization required as response to high Toll signals at the ventral side of the embryo. Furthermore, N-terminal deleted Cactus mimics these effects, indicating that the ability of Cactus to favor Toll stems from mobilization of a free Cactus pool induced by the Calpain A protease. These results indicate that unexplored mechanisms are at play to ensure a correct response to high Toll signals. 


\section{Introduction}

The evolutionarily conserved Toll receptor pathway is implicated in the control of development, proliferation, and immunity. Toll signals are modulated at many levels, characteristic that warrants an assortment of possible outcomes (Mitchell et al., 2016). To understand Toll pathway architecture it is necessary to quantitatively define how each pathway element contributes to the final response. Inhibitor of $\mathrm{NF \kappa B}(\mathrm{I} \kappa \mathrm{B})$ proteins comprise the Toll responsive complex together with NFkB effectors. Therefore, they are central elements of the Toll pathway that require careful analysis of their effects.

Drosophila is a unique system to identify how IאB proteins tune Toll responses as disturbances in IKB function can be analyzed concomitantly across a range of Toll activation levels. During embryogenesis, ventral-lateral activation of maternal Toll receptors leads to a ventral-to-dorsal nuclear gradient of the NFKB/CRel protein Dorsal (Dl). Inside the nucleus, differential affinity of Dl-target genes results in spatial control of gene expression along the dorsal-ventral (DV) axis (Rushlow and Shvartsman, 2012). High Toll signals lead to ventral mesodermal gene expression, intermediate signals induce lateral neuroectodermal genes, while low signals allow dorsal ectodermal gene expression. Toll signals are transduced through mobilization of adaptor proteins, protein kinase activation, and ultimately phosphorylation and proteasomal degradation of the sole Drosophila IкB protein Cactus. Cactus (Cact) degradation then exposes nuclear localization sequences in $\mathrm{Dl}$ and nuclear translocation ensues (Stein and Stevens, 2014).

The amount of nuclear Dorsal (nDl) is used as readout for the level of Toll pathway activation. Quantitative analysis of $\mathrm{nDl}$ in fixed and live embryos has shown that the $\mathrm{Dl}$ gradient is visible since early blastoderm cycle 9, increasing in amplitude with time until mitotic cycle 14 (Kanodia et al., 2009; Liberman et al., 2009; Reeves et al., 2012). Besides a deep understanding of the dynamics of the Dl gradient, how cactus affects this gradient has not been investigated. Using quantitative analysis we show that loss of cactus flattens the nDl gradient, implying 
that cactus favors Dorsal nuclear localization in addition to its widely established role to inhibit Toll signals.

\section{Results and Discussion}

\section{Cactus favors Dorsal responses to high Toll signals}

In order to identify how Cact tunes $N F \kappa B$ responses we investigated the effects of reducing cactus on Dl nuclear localization and expression of Dl target genes. It was previously reported that the $\mathrm{nDl}$ gradient expands, and the ventral twist (twi) expression domain widens in embryos generated from mothers carrying cact loss-of-function germline clones (Roth et al., 1991). This effect results from the near absence of Cact protein, which releases $\mathrm{Dl}$ inhibition in the cytoplasm. However, smaller reductions in cact lead to a range of DV embryonic phenotypes (Govind et al., 1993; Isoda and Nusslein-Volhard, 1994; Roth et al., 1991).

Here we investigate the effect of reducing, but not completely depriving the embryo of the Cact inhibitor. We progressively decreased maternal Cact protein to $50 \%$ wild type levels (Fig. 1 and Fig. S1). Reducing the cact gene dose by half leads to a minimal reduction in Cact protein and induces 30\% embryo lethality (cact[A2] heterozygotes, (Govind et al., 1993), with no detectable DV embryonic phenotype (Fig. 1B,E,F,I,J and Table SI). Further Cact reductions, however, result in consistent alterations along the DV axis.

In agreement with previous reports, decreasing Cact increases $\mathrm{nDl}$ in regions of the embryo that receive intermediate or low Toll signals (Fig. 1B-D): A $30 \%$ reduction in Cact protein is sufficient to expand lateral nDl levels to dorsal regions of the embryo (Fig 1C). A 40\% reduction in Cact has the same effect, but additionally reduces $\mathrm{nDl}$ in the ventral domain (Fig. 1D). These changes in $\mathrm{nDl}$ are reflected on the pattern of Dl target genes. The lateral domain of short gastrulation (sog) expression, a gene that requires intermediate levels of $\mathrm{nDl}$ for activation (Stathopoulos and Levine, 2002), expands as Cact levels decrease by 30\% or more 
(Fig. 1G,H,K,L). In the ventral prospective mesoderm the domains of snail (sna) and twi expression, genes that require high levels of nDl for activation (Ip et al., 1991; Papatsenko and Levine, 2005), are reduced as Cact levels drop (Fig. 1G,H,L and N,O and Fig. S2). Therefore, we find that Cact performs an additional function to favor Toll signals, distinct from its established function to inhibit Dl nuclear translocation. Importantly, this positive effect is restricted to regions that withstand high levels of Toll signaling.

Next, we investigated whether this positive effect depends on Dl. First, we reasoned that the amount of $\mathrm{Dl}$ protein might be limiting in contexts where Toll signals are high. In fact, in embryos laid by $d l$ - heterozygous mothers $\mathrm{nDl}$ and the ventral sna domain narrow compared to controls (Fig. 2A,C) (Fontenele et al., 2013; Kanodia et al., 2009). Therefore, decreasing $d l$ should sensitize the embryo to Cact reductions if the positive role of Cact depends on Dl. In agreement with this hypothesis, a minimal reduction in Cact (as in cact[A2] heterozygotes) further decreases $\mathrm{nDl}$ along the ventral and lateral domains of the embryo (Fig. 2B,D), an effect not observed in a wild type background (Fig. 1B). As a consequence of this reduction in Cact, the lateral sog domain expands into the ventral, high Toll activity, domain (Fig. 2C-H). These results confirm that cact favors Toll signals, and show that this effect is dependent on the amount of $\mathrm{Dl}$.

\section{Mobilization of free Cactus promotes Toll signals}

Since decreasing cact lead to loss of ventral $\mathrm{nDl}$, we asked whether we could detect a corresponding activity by overexpressing Cact constructs under the control of a maternal promoter (CaM,(Fernandez et al., 2001). Importantly, $\mathrm{CaM}>$ cact-eGFP perfectly recovers the $\mathrm{nDl}$ gradient in a cact[A2]/cact[011] loss-offunction background (Fig. S3A, compare to Fig. 1D), implying that maternal induction of cact-eGFP mimics wild type Cact behavior.

We tested the effects of two Cact constructs on the $\mathrm{nDl}$ gradient: full-length and N-terminal truncated Cact-eGFP. Two pathways control Cact levels: Cact is phosphorylated at $\mathrm{N}$-terminal serine residues and degraded through the 
proteasome in response to Toll pathway activation (Bergmann et al., 1996; Fernandez et al., 2001; Hecht and Anderson, 1993; Reach et al., 1996; Shelton and Wasserman, 1993). Cact is also subject to C-terminal CKII kinase phosphorylation through a Toll independent pathway (Belvin et al., 1995; Liu et al., 1997; Packman et al., 1997). N-terminal truncated Cact mutants (cact[E10] and $\operatorname{cact}[\mathrm{BQ}])$ are irresponsive to Toll (Bergmann et al., 1996; Roth, 2001). Interestingly, N-terminal truncated Cact, hereafter referred to as Cact[E10], is produced endogenously from full-length Cact as a Calpain A protease cleavage product. Both full-length Cact and Cact[E10] bind Dl (Fontenele et al., 2013).

Maternal expression of Cact-eGFP and Cact[E10]-eGFP in cact loss-offunction backgrounds reveals that Cact[E10] favors Toll. When endogenous Cact is $30 \%$ reduced, Cact-eGFP recovers $\mathrm{nDl}$ and reduces lateral sog to a wild type pattern (Fig. S3A,F,I). Therefore, Cact-eGFP inhibits the expression of a Toll pathway target, as expected. The same level of Cact-eGFP expression has no effect once endogenous Cact is 50\% reduced (Fig. 3A,C,D,F and G,HF,). Strikingly, Cact[E10]-eGFP increases the ventral sna domain and decreases the lateral sog domain in the same condition (Fig. 3A,B,D,E and G,H), although it has no effect when endogenous Cact is only 30\% reduced (Fig. S3). Therefore, Cact[E10]-eGFP favors Toll in the ventral, high-Toll signaling domain and inhibits intermediate Toll signals in the lateral domain. In addition, this effect depends on the relative amount of endogenous full-length Cact.

The finding that Cact[E10] favors Toll is surprising considering that the gain-of-function cact[E10] allele inhibits Toll (Bergmann et al., 1996; Govind et al., 1993). Our results confirm that Cact[E10] inhibits Toll since decreases the lateral sog domain, target of intermediate Toll. However, Cact[E10] additionally favors Toll, depending on the respective amount of full-length Cact. Since N-terminal truncated Cact is produced endogenously (Fontenele et al., 2013), we conclude that Cact[E10] harbors the positive effect we have detected in the loss-of-function assays shown above.

The ability of Cact[E10]-eGFP to alter Toll signals depends on the amount of Dl. Cact-eGFP and Cact[E10]-eGFP produce a comparable decrease in $\mathrm{nDl}$ in a $d l$ heterozygous background (Fig. S4 compare to Fig. 2A), although Cact[E10]-eGFP 
has no effect in the presence of wild type Dl levels. Together with the results described above, this suggests that Cact[E10] competes with wild type Cact to bind $\mathrm{Dl}$, and indicates the existence of a delicate balance between levels of full-length Cact, truncated Cact, and Dl for proper Toll signaling events.

In Drosophila, Cact partitions between free and NFKB-bound complexes. Dlbound Cact comprises the Toll responsive complex (1Cact:2Dl), while Dl-free Cact (2Cact) is target of a Toll independent pathway (Bergmann et al., 1996; Liu et al., 1997). We have shown that the Calpain A protease, which leads to the production of Cact[E10], targets only Dl-free Cact. Calpain $A$ knockdown decreases nDl in ventral regions, which implies that Calpain A favors Toll signals (Fontenele et al., 2013). Based on these findings, our results point to a model where Cact that is unbound to $\mathrm{Dl}$ (free Cact) is released by the action of Calpain A to favor Toll signals in ventral regions of the Drosophila embryo (Fig. 4). Interestingly, vertebrate Calpains also target $\mathrm{I} \kappa \mathrm{B}$ proteins and the pathways that control free and NFKBbound Cactus and IкB $\alpha$ are conserved (Han et al., 1999; Li et al., 2010; Pando and Verma, 2000; Schaecher et al., 2004; Shen et al., 2001; Shumway et al., 1999). Therefore, our findings of a positive function for Cactus may have important implications for the control of vertebrate Toll signals.

\section{On the nature of the positive function exerted by Cactus}

Mathematical modeling pointed out the importance of IкB proteins in modulating Toll signals in vertebrates and in Drosophila sp. (Ambrosi et al., 2014; Kearns and Hoffmann, 2009; O'Dea et al., 2007). Particularly, it was shown that free $\mathrm{I} \kappa \mathrm{B}$ is an important regulatory target for the control of Toll signals (Konrath et al., 2014). Our previous analyses suggested that Drosophila Calpain A mobilizes free Cact to replenish limiting amounts of Cact:2Dl complexes for Toll signaling. With the present finding that Cact favors Toll, and that Cact[E10], a product of Calpain A activity, harbors this function, we now have quantitative data to support this hypothesis. Different mechanisms involving Cact[E10] could potentially favor Toll. 
For instance, Toll signals depend on the mobilization of pre-signaling complexes (Marek and Kagan, 2012; Sun et al., 2004), endocytosis (Huang et al., 2010; Lund et al., 2010), and how long NFKB proteins remain bound to DNA (Mitchell et al., 2016; O'Connell and Reeves, 2015). Thus, mechanisms involving Cact[E10] that favor the formation of pre-signaling complexes, the mobilization of endocytic vesicles containing Toll pathway elements or that alter the resident time of $\mathrm{Dl}$ in the nucleus, may positively impact Toll signals and explain our results. Furthermore, it has been recently proposed that Cact may function by a shuttling mechanism to concentrate Dl to the ventral side of the Drosophila embryo (Carrell et al., 2016) preprint), akin to the shuttling mechanism exerted by the BMP inhibitor Sog to shuttle and concentrate BMPs to the dorsal side of the embryo (Mizutani et al., 2005; Shimmi et al., 2005; Umulis et al., 2006). While further research is required to understand how Cact enhances nDl levels in ventral regions of the embryo and consequently Dl-target gene expression, we have clearly uncovered a novel function for the Cact inhibitor to favor Toll. 


\section{Methods}

\section{Fly stocks and genetic crosses}

Lines used in this study were: loss-of-function cact[A2] and cact [011], generously provided by Steve Wasserman, $d l[6]$ and cact[A4] obtained from the Bloomington Indiana Stock Center. Df(2L)cact[255]/CyO was used as a cact deficiency and is here denoted as Df(cact). Maternal overexpression lines were CaM>cactus-eGFP and CaM>cactus[E10]-eGFP as described in (Fontenele et al., 2013). All embryos were collected from mothers of the respective genotypes crossed to wild type Canton S males.

\section{Immunoblotting}

Bleach dechorionated 30min-1h30min old embryos of the appropriate genotypes were homogenized in lysis buffer $(1$ embryo/ $\mu \mathrm{l})$ and prepared for SDS-PAGE as in (Fontenele et al., 2009). Endogenous Cactus and Dorsal were detected with monoclonal antibodies from Developmental Studies Hybridoma Bank (DSHB, antiDl 1:100 and anti-Cact 1:500). Anti- $\alpha$ Tubulin was used as loading control (DM1 $\alpha$; 1:3000; Sigma). Full length and truncated Cact-eGFP were detected using an antiGFP polyclonal antiserum (1:1000; Novus Biologicals).

\section{Immunohistochemistry an in situ hybridization}

For visualization of the $\mathrm{nDl}$ gradient mutant and control Histone-GFP embryos were mixed, fixed and processed concomitantly as in (Fontenele et al., 2013). Primary antisera used were monoclonal anti-Dl (7A4; 1:100; DSHB) and anti-GFP (1:1000; Novus Biologicals) to detect control gradients. Dl target genes were visualized by in situ hybridization as in (Fontenele et al., 2009).

\section{Quantitative analysis}

Images of the $\mathrm{nDl}$ gradient and $\mathrm{Dl}$ target genes were collected from mid stage 14 embryos, as defined by the amount of membrane invagination around nuclei. Quantification of the Dl gradient was as described in (Kanodia et al., 2009) using 
Matlab, collected at 85\% egg length using a microfluidic device to orient embryos for ends-on imaging (Chung et al., 2011). For upright imaging, a Nikon 60× PlanApo oil objective was used, and images were collected at the focal plane $\sim 90 \mu \mathrm{m}$ from the anterior pole of the embryo. For the overall effect on Dl target genes, embryos were imaged laterally. All genotypes were processed and analyzed in parallel, thus the same wild type control is shown in graphs. Images were acquired with a Nikon A1 or a Leica LSM confocal microscope.

\section{Statistical analysis}

Student's $t$-test was performed for all experiments. Results are displayed as mean $\pm \mathrm{SEM}$. The level of significance is shown in each figure $(* * * \mathrm{P} \leq 0.001, * * \mathrm{P} \leq 0.01$, $* * \mathrm{P} \leq 0.05)$,

\section{Acknowledgements}

We thank Eric Wieschaus for the anti-twist antiserum. Monoclonal antibodies anti$\mathrm{Dl}$ and anti-Cact originally developed by Ruth Steward were obtained from the Developmental Studies Hybridoma Bank, created by the NICHD of the NIH and maintained at The University of Iowa, Department of Biology, Iowa City, IA 52242.

\section{Competing interests}

The authors declare that they have no conflict of interest.

\section{Author contributions}

MAC, and MF performed experiments. MAC, MF and BO analyzed the data. MAC, PMB, SS and HMA developed the approach and prepared or edited the manuscript.

\section{Funding}


This research was funded by the Brazilian Cientific Council (CNPq-Brazil, 477157/2013-0). MAC was a recipient of CAPES/Brazil local and overseas fellowships. 


\section{Figure Legends}

Figure 1. Cactus inhibits Toll in lateral regions and favors Toll signals in ventral regions of the embryo. A) Optical section of a control Dl gradient. B-D) Nuclear Dl levels were extracted and plotted as half gradients for control embryos (black) or embryos from $\operatorname{cact}[\mathrm{A} 2] /+$ (B), $\operatorname{cact}[\mathrm{A} 2] / \operatorname{cact}[011]$ (C) or $\operatorname{cact}[\mathrm{A} 2] / \mathrm{Df}(\mathrm{cact}) \quad$ (D) mothers (red). Y axis represents fluorescence intensity of $\mathrm{nDl}$ along the ventral-to-dorsal embryonic axis (X axis). (E-L) in situ hybridization for sna (green) in the mesoderm and sog (pink) in the lateral neuroectoderm of embryos with maternal cactus reductions. In embryos from cact heterozygotes no change in the ventral and lateral territories are observed $(\mathrm{F}, \mathrm{J})$. Further reductions lead to a dorsal expansion of lateral sog and reduction in the ventral prospective mesoderm $(\mathrm{G}, \mathrm{H}, \mathrm{K}, \mathrm{L})$. Anterior is left, posterior right, dorsal up in $G$ to $L$. Percentage in upper right indicates the average amount of Cactus protein relative to wild type, defined by quantification of protein levels in western blots ( $\mathrm{M}$ and Suppl. Fig.1). (N,O) Width of the snail domain measured at 75\% and 50\% egg length. Statistically significant differences based on Student's t-test, displayed as mean \pm s.e.m. $(* * * \mathrm{P} \leq 0.001, * * \mathrm{P} \leq 0.01, * \mathrm{P} \leq 0.05)$.

Figure 2. Cactus ability to favor Toll signals depends on the amount of Dorsal. A,B) nDl gradients for control embryos (black) or embryos from $d l[6] /+(A)$, or $d l[6] / \operatorname{cact}[\mathrm{A} 2](\mathrm{B})$ mothers (red). The $\mathrm{nDl}$ gradient decreases by reducing maternal $d l$ and cact, compared to control embryos stained concomitantly. (C-F) in situ hybridization and $(\mathrm{G}, \mathrm{H})$ quantification of sna (green) and sog (pink) in control (E) and maternal $d l[6] / \operatorname{cact}[\mathrm{A} 2]$ (F) transverse sections. Coincident sog and sna expression is seen in ventral regions (F). The size of the sog domain includes both left and right sides of the embryo since in $d l[6] / \operatorname{cact}[\mathrm{A} 2]$ this 
domain is frequently continuous. Statistically significant differences based on Student's t-test, displayed as mean \pm s.e.m. (***P $\leq 0.001, * \mathrm{P} \leq 0.05)$.

Figure 3. The Cact[E10] fragment favors Toll. (A-F) in situ hybridization for sna and sog in cact[A2]/Df(cact) (A,D); cact[A2]/Df(cact); cactE10-eGFP/+ $(\mathrm{B}, \mathrm{E})$ and cact $[\mathrm{A} 2] / \mathrm{Df}($ cact $)$; cact-eGFP/+ (C,F). (G,H) Quantification of sna at 75\% egg length (G) and sog (H) domains. Full dorsal sog expansion is defined when the domain is greater that 25 cells and no dorsal zen expression is observed. Statistically significant differences based on Student's t-test, displayed as mean \pm s.e.m. ( $\left.{ }^{* * *} \mathrm{P} \leq 0.001\right)$.

Figure 4. Model for free Cact function. A. $\mathrm{nDl}$ and ventral gene domains are reduced when Cact is decreased. B. This suggests that free Cact favors the formation of Dl/Cact complexes required for high Toll signals. 


\section{References}

Ambrosi, P., Chahda, J. S., Koslen, H. R., Chiel, H. J. and Mizutani, C. M. (2014). Modeling of the dorsal gradient across species reveals interaction between embryo morphology and Toll signaling pathway during evolution. PLoS Comput Biol 10, e1003807.

Belvin, M. P., Jin, Y. and Anderson, K. V. (1995). Cactus protein degradation mediates Drosophila dorsal-ventral signaling. Genes \& development 9, 783793.

Bergmann, A., Stein, D., Geisler, R., Hagenmaier, S., Schmid, B., Fernandez, N., Schnell, B. and Nusslein-Volhard, C. (1996). A gradient of cytoplasmic Cactus degradation establishes the nuclear localization gradient of the dorsal morphogen in Drosophila. Mechanisms of development 60, 109-123.

Carrell, S. N., O'Connell, M. D., Allen, A. E., Smith, S. M. and Reeves, G. T. (2016). A Facilitated Diffusion Mechanism Establishes the Drosophila Dorsal Gradient. bioRxivdoi: http://dx.doi.org/10.1101/057091

Chung, K., Kim, Y., Kanodia, J. S., Gong, E., Shvartsman, S. Y. and Lu, H. (2011). A microfluidic array for large-scale ordering and orientation of embryos. Nature methods 8, 171-176.

Fernandez, N. Q., Grosshans, J., Goltz, J. S. and Stein, D. (2001). Separable and redundant regulatory determinants in Cactus mediate its dorsal group dependent degradation. Development 128, 2963-2974.

Fontenele, M., Carneiro, K., Agrellos, R., Oliveira, D., Oliveira-Silva, A., Vieira, V., Negreiros, E., Machado, E. and Araujo, H. (2009). The Ca2+-dependent protease Calpain A regulates Cactus/I kappaB levels during Drosophila development in response to maternal Dpp signals. Mechanisms of development 126, 737-751.

Fontenele, M., Lim, B., Oliveira, D., Buffolo, M., Perlman, D. H., Schupbach, T. and Araujo, H. (2013). Calpain A modulates Toll responses by limited Cactus/IkappaB proteolysis. Molecular biology of the cell 24, 2966-2980.

Govind, S., Brennan, L. and Steward, R. (1993). Homeostatic balance between dorsal and cactus proteins in the Drosophila embryo. Development 117, 135-148.

Han, Y., Weinman, S., Boldogh, I., Walker, R. K. and Brasier, A. R. (1999). Tumor necrosis factor-alpha-inducible IkappaBalpha proteolysis mediated by cytosolic m-calpain. A mechanism parallel to the ubiquitin-proteasome pathway for nuclear factor-kappab activation. The Journal of biological chemistry 274, 787-794.

Hecht, P. M. and Anderson, K. V. (1993). Genetic characterization of tube and pelle, genes required for signaling between Toll and dorsal in the specification of the dorsal-ventral pattern of the Drosophila embryo. Genetics 135, 405-417.

Huang, H. R., Chen, Z. J., Kunes, S., Chang, G. D. and Maniatis, T. (2010). Endocytic pathway is required for Drosophila Toll innate immune signaling. 
Proceedings of the National Academy of Sciences of the United States of America 107, 8322-8327.

Ip, Y. T., Kraut, R., Levine, M. and Rushlow, C. A. (1991). The dorsal morphogen is a sequence-specific DNA-binding protein that interacts with a long-range repression element in Drosophila. Cell 64, 439-446.

Isoda, K. and Nusslein-Volhard, C. (1994). Disulfide cross-linking in crude embryonic lysates reveals three complexes of the Drosophila morphogen dorsal and its inhibitor cactus. Proceedings of the National Academy of Sciences of the United States of America 91, 5350-5354.

Kanodia, J. S., Rikhy, R., Kim, Y., Lund, V. K., DeLotto, R., Lippincott-Schwartz, J. and Shvartsman, S. Y. (2009). Dynamics of the Dorsal morphogen gradient. Proceedings of the National Academy of Sciences of the United States of America 106, 21707-21712.

Kearns, J. D. and Hoffmann, A. (2009). Integrating computational and biochemical studies to explore mechanisms in NF-\{kappa\}B signaling. The Journal of biological chemistry 284, 5439-5443.

Konrath, F., Witt, J., Sauter, T. and Kulms, D. (2014). Identification of new IkappaBalpha complexes by an iterative experimental and mathematical modeling approach. PLoS Comput Biol 10, e1003528.

Li, C., Chen, S., Yue, P., Deng, X., Lonial, S., Khuri, F. R. and Sun, S. Y. (2010). Proteasome inhibitor PS-341 (bortezomib) induces calpain-dependent IkappaB(alpha) degradation. The Journal of biological chemistry 285, 1609616104.

Liberman, L. M., Reeves, G. T. and Stathopoulos, A. (2009). Quantitative imaging of the Dorsal nuclear gradient reveals limitations to threshold-dependent patterning in Drosophila. Proceedings of the National Academy of Sciences of the United States of America 106, 22317-22322.

Liu, Z. P., Galindo, R. L. and Wasserman, S. A. (1997). A role for CKII phosphorylation of the cactus PEST domain in dorsoventral patterning of the Drosophila embryo. Genes \& development 11, 3413-3422.

Lund, V. K., DeLotto, Y. and DeLotto, R. (2010). Endocytosis is required for Toll signaling and shaping of the Dorsal/NF-kappaB morphogen gradient during Drosophila embryogenesis. Proceedings of the National Academy of Sciences of the United States of America 107, 18028-18033.

Marek, L. R. and Kagan, J. C. (2012). Phosphoinositide binding by the Toll adaptor dMyD88 controls antibacterial responses in Drosophila. Immunity 36, 612622.

Mitchell, S., Vargas, J. and Hoffmann, A. (2016). Signaling via the NFkappaB system. Wiley Interdiscip Rev Syst Biol Med 8, 227-241.

Mizutani, C. M., Nie, Q., Wan, F. Y., Zhang, Y. T., Vilmos, P., Sousa-Neves, R., Bier, E., Marsh, J. L. and Lander, A. D. (2005). Formation of the BMP activity gradient in the Drosophila embryo. Developmental cell 8, 915-924.

O'Connell, M. D. and Reeves, G. T. (2015). The presence of nuclear cactus in the early Drosophila embryo may extend the dynamic range of the dorsal gradient. PLoS Comput Biol 11, e1004159. 
O'Dea, E. L., Barken, D., Peralta, R. Q., Tran, K. T., Werner, S. L., Kearns, J. D., Levchenko, A. and Hoffmann, A. (2007). A homeostatic model of IkappaB metabolism to control constitutive NF-kappaB activity. Molecular systems biology 3, 111.

Packman, L. C., Kubota, K., Parker, J. and Gay, N. J. (1997). Casein kinase II phosphorylates Ser468 in the PEST domain of the Drosophila IkappaB homologue cactus. FEBS letters 400, 45-50.

Pando, M. P. and Verma, I. M. (2000). Signal-dependent and -independent degradation of free and NF-kappa B-bound IkappaBalpha. The Journal of biological chemistry 275, 21278-21286.

Papatsenko, D. and Levine, M. (2005). Quantitative analysis of binding motifs mediating diverse spatial readouts of the Dorsal gradient in the Drosophila embryo. Proceedings of the National Academy of Sciences of the United States of America 102, 4966-4971.

Reach, M., Galindo, R. L., Towb, P., Allen, J. L., Karin, M. and Wasserman, S. A. (1996). A gradient of cactus protein degradation establishes dorsoventral polarity in the Drosophila embryo. Developmental biology 180, 353-364.

Reeves, G. T., Trisnadi, N., Truong, T. V., Nahmad, M., Katz, S. and Stathopoulos, A. (2012). Dorsal-ventral gene expression in the Drosophila embryo reflects the dynamics and precision of the dorsal nuclear gradient. Developmental cell 22, 544-557.

Roth, S. (2001). Drosophila oogenesis: coordinating germ line and soma. Current biology : CB 11, R779-781.

Roth, S., Hiromi, Y., Godt, D. and Nusslein-Volhard, C. (1991). cactus, a maternal gene required for proper formation of the dorsoventral morphogen gradient in Drosophila embryos. Development 112, 371-388.

Rushlow, C. A. and Shvartsman, S. Y. (2012). Temporal dynamics, spatial range, and transcriptional interpretation of the Dorsal morphogen gradient. Curr Opin Genet Dev 22, 542-546.

Schaecher, K., Goust, J. M. and Banik, N. L. (2004). The effects of calpain inhibition on IkB alpha degradation after activation of PBMCs: identification of the calpain cleavage sites. Neurochemical research 29, 1443-1451.

Shelton, C. A. and Wasserman, S. A. (1993). pelle encodes a protein kinase required to establish dorsoventral polarity in the Drosophila embryo. Cell 72, 515-525.

Shen, J., Channavajhala, P., Seldin, D. C. and Sonenshein, G. E. (2001). Phosphorylation by the protein kinase CK2 promotes calpain-mediated degradation of IkappaBalpha. J Immunol 167, 4919-4925.

Shimmi, O., Umulis, D., Othmer, H. and O'Connor, M. B. (2005). Facilitated transport of a Dpp/Scw heterodimer by Sog/Tsg leads to robust patterning of the Drosophila blastoderm embryo. Cell 120, 873-886.

Shumway, S. D., Maki, M. and Miyamoto, S. (1999). The PEST domain of IkappaBalpha is necessary and sufficient for in vitro degradation by mucalpain. The Journal of biological chemistry 274, 30874-30881.

Stathopoulos, A. and Levine, M. (2002). Dorsal gradient networks in the Drosophila embryo. Developmental biology 246, 57-67. 
Stein, D. S. and Stevens, L. M. (2014). Maternal control of the Drosophila dorsalventral body axis. Wiley Interdiscip Rev Dev Biol 3, 301-330.

Sun, H., Towb, P., Chiem, D. N., Foster, B. A. and Wasserman, S. A. (2004). Regulated assembly of the Toll signaling complex drives Drosophila dorsoventral patterning. The EMBO journal 23, 100-110.

Umulis, D. M., Serpe, M., O'Connor, M. B. and Othmer, H. G. (2006). Robust, bistable patterning of the dorsal surface of the Drosophila embryo. Proceedings of the National Academy of Sciences of the United States of America 103, 11613-11618. 


\section{Cardoso et al. Figure 1}
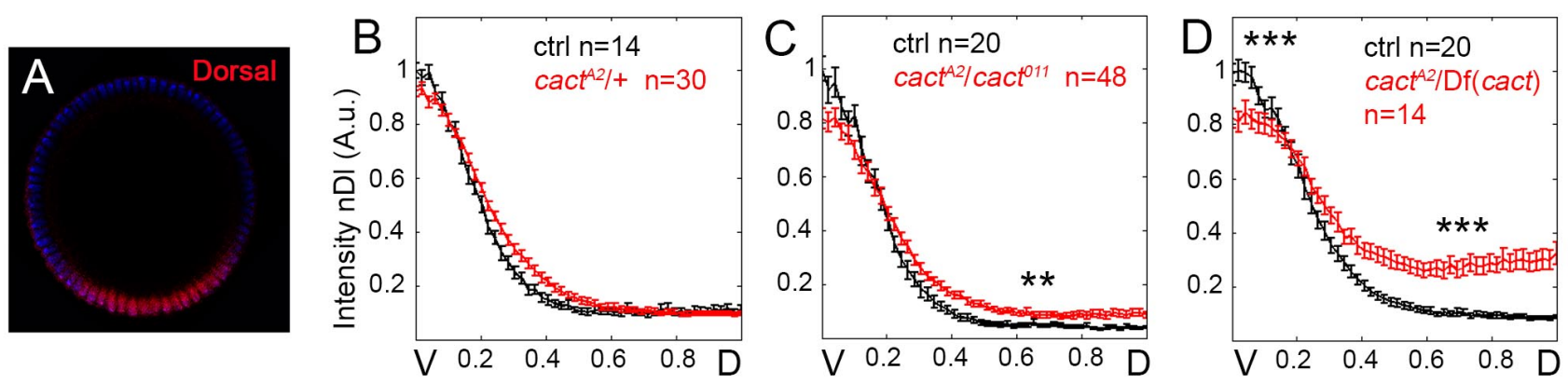

more Cactus

less Cactus
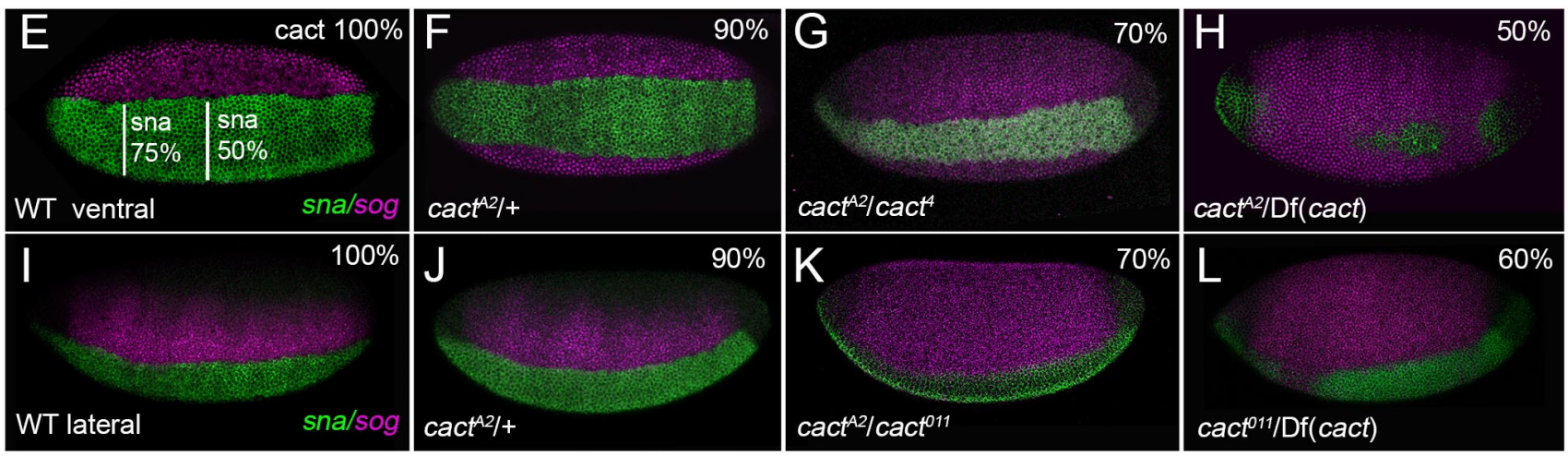

M

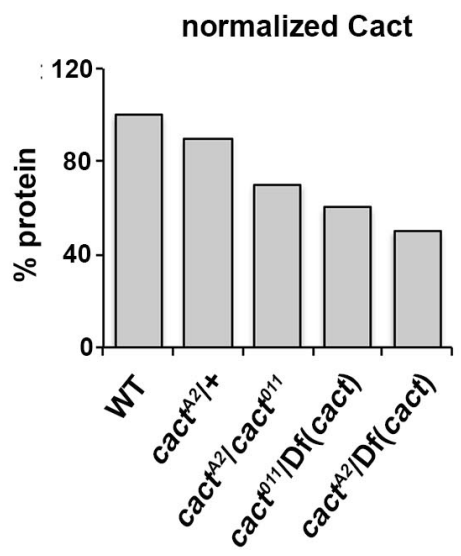

$\mathrm{N}$

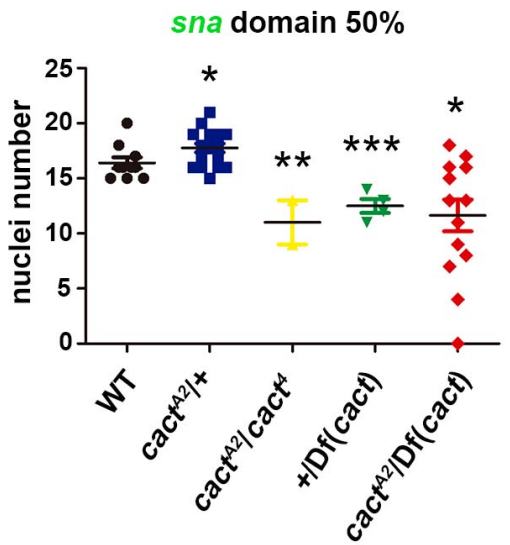

$\mathrm{O}$

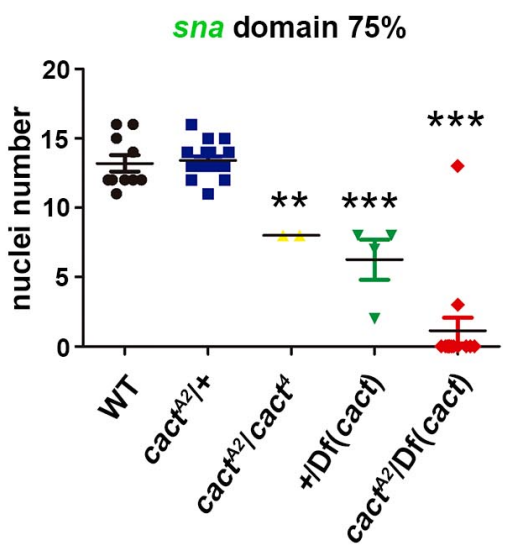


Cardoso et al. Figure 2
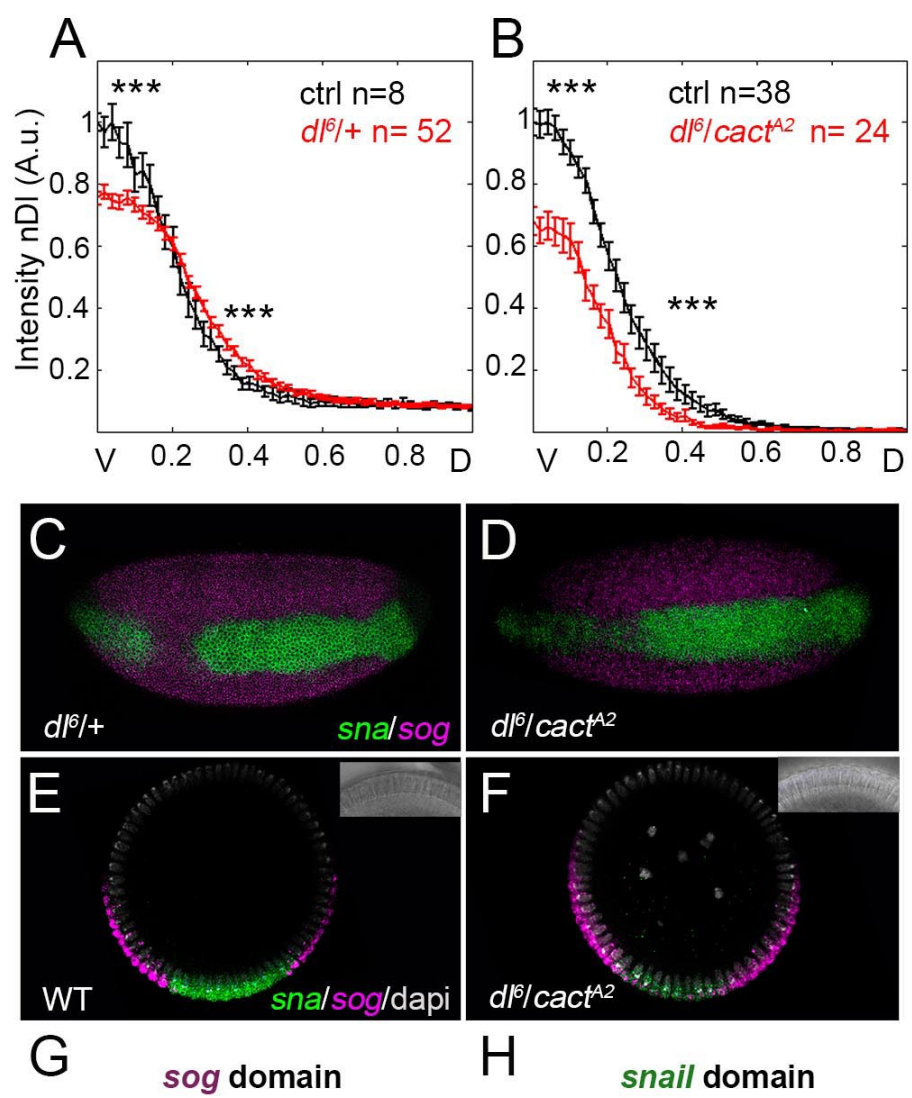

$\mathrm{H}$ snail domain
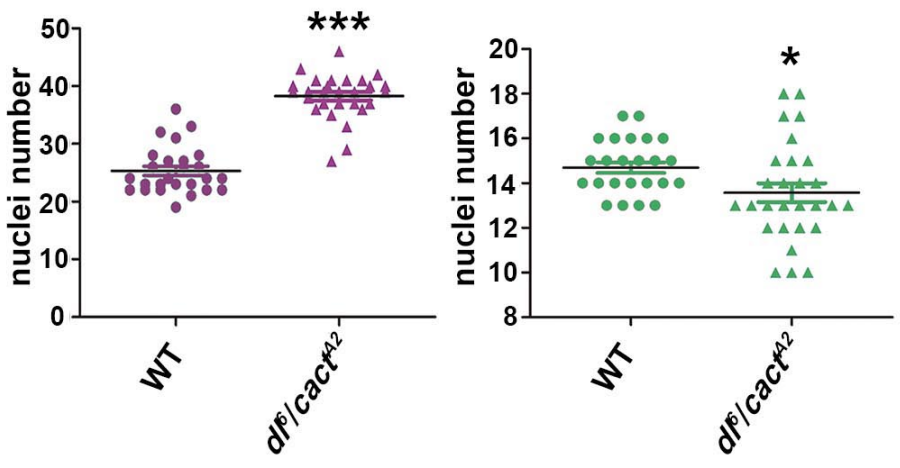


\section{Cardoso et al. Figure 3}

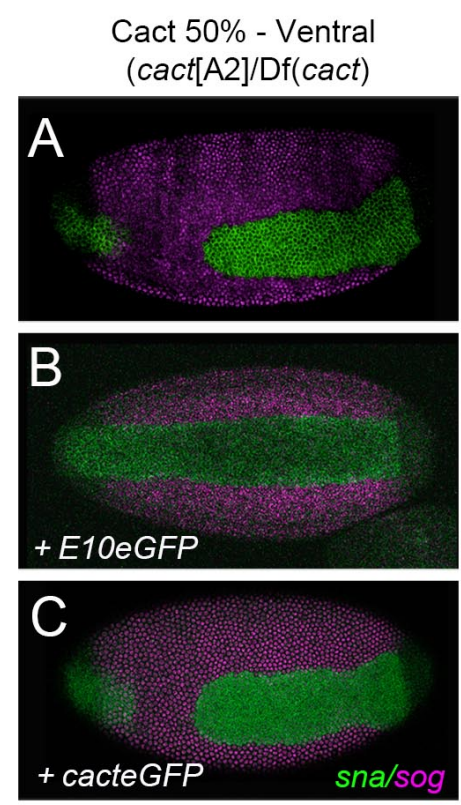

G
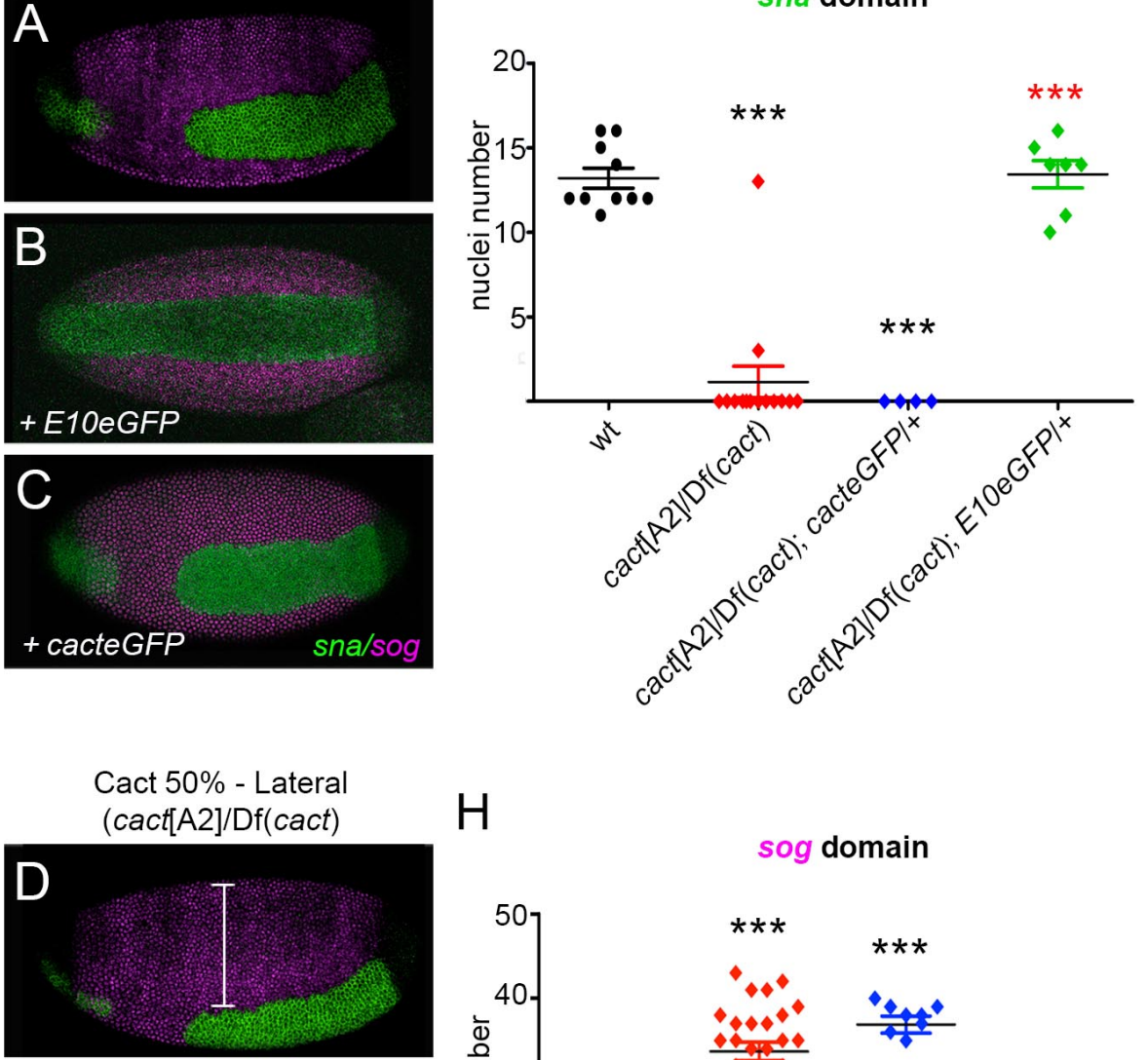

$\mathrm{H}$
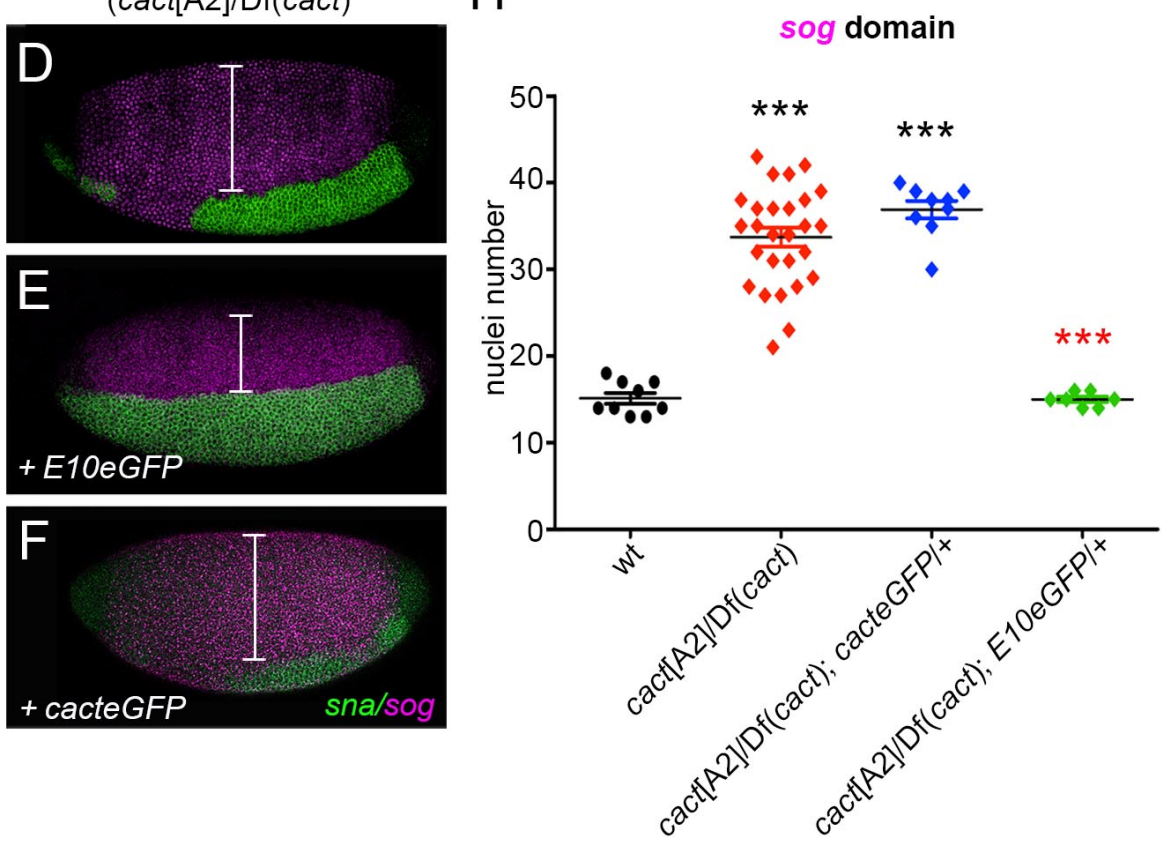


\section{Cardoso et al. Figure 4}
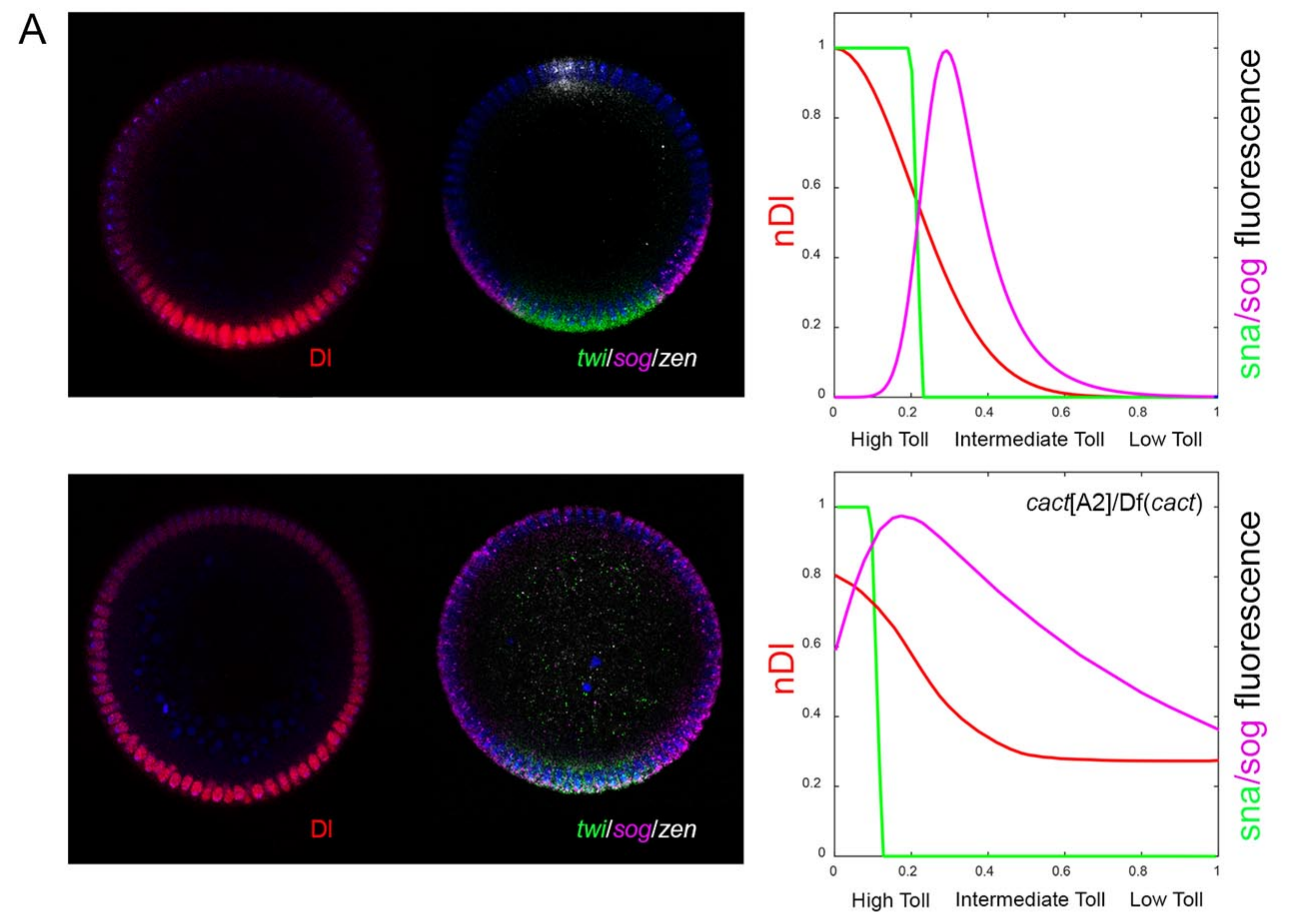

\section{B}

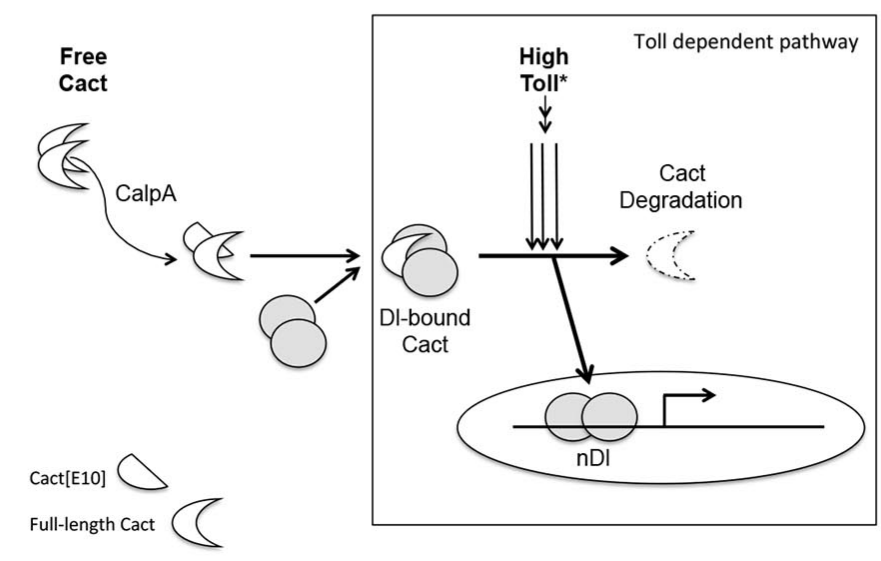

